\title{
Experimental and numerical analysis of size effects on stress intensity in anisotropic porous materials
}

\author{
Dimitra Touliatou, Marcus A. Wheel \\ Department of Mechanical and Aerospace Engineering \\ University of Strathclyde, Glasgow, G1 1XJ, United Kingdom
}

\begin{abstract}
A prominent size effect has previously been reported for the fracture behaviour of brittle porous materials, with smaller specimens behaving quite differently to their larger counterparts. In such materials, the size of the K-dominant zone has been numerically found to be greatly affected by the presence of voids in the near-tip area, thus putting the assumption of a single fracture parameter under question. In order to address this, in this study mode I tests are conducted on porous double cantilever beam specimens, while the stress distribution in the near-tip area is being observed by means of photoelasticity. Results validate the predicted size effect and suggest that the voids can indeed alter the size and shape of the stress pattern in the specimens. A parametric study is then conducted to investigate the influence of void shape variations that can be caused by manufacturing inaccuracies on the stress concentration at the crack tip. It is found that although the stress intensity at the crack tip can be greatly affected by such factors, the size of the K-dominant zone remains unaffected.
\end{abstract}

Keywords: Fracture mechanics, Size effect, Porous materials, Experimental, Cell shape, Non-singular stresses

Email address: marcus. wheel@strath.ac.uk (Marcus A. Wheel) 


\section{Introduction}

Material characterisation through standardised testing involves several specimens of specified dimensions in controlled conditions to extract repeatable, consistent results regarding a material's property. However, the extrapolation of a material's estimated property from a predefined specimen to a generic geometry and size component is not always evident.

A number of theories attempt to resolve these discrepancies. Initially, any inconsistencies in the scaling of a material's strength were mostly attributed to its stochastic distribution and the statistically increasing presence of flaws with increased size, as described by the Weibull distribution [1]. Later experiments in heterogeneous materials (e.g. [2-4] for concrete, [5-7] for polymer foams, [8, 9] for composites), showed that the exhibited size effect can instead be attributed to the material's transition to plasticity in small scales. A new strength scaling theory was established, suggesting that when the size of the heterogeneity becomes comparable to the specimen's significant dimensions, the fracture process zone prevails and linear elastic fracture mechanics (LEFM) no longer applies. However, the heterogeneous materials presented in these studies are not purely brittle but quasi-brittle, exhibiting softening effects and other energy dissipating micro-phenomena around the crack tip.

Small scale yielding (SSY) is a central concept of LEFM. Based on the assumption of SSY, the fracture process zone exhibited in brittle materials is very small, implying that any non-linear phenomena are negligible [10]. In turn, it is assumed that there exists a relatively large zone, the K-dominant zone, inside which it is considered that the near-tip stress field can be accurately described by employing a single parameter, the stress intensity factor (SIF), the critical value of which is constant for each material [11].

Experiments on brittle materials are mainly focused on homogeneous specimens. However, even for this presumably predictable case, different values of fracture toughness have been reported for different specimen configurations or sizes, deviating from the usual scaling predicted by LEFM [12-16]. In order to explain such discrepancies, scientists are rejecting the notion of an indisputably large K-dominant zone and are investigating two parameter models, including non-singular terms in addition to the SIF, to more accurately and consistently predict the fracture toughness of different specimens.

Two-parameter models that include the second, non-singular term of the William's equation [17], the T-stress, are the most widely investigated 
$[16,18-22]$. Results show that using such models can more accurately predict the behaviour in a variety of materials for mode I, II and mixed modes of fracture. Some studies on the mode I behaviour of brittle materials have alternatively included the third term of the William's equation, $A_{1 / 2}$, which is also non-singular. Again, accuracy in the predictions of the material's behaviour increased $[14,23,24]$ even in the case of heterogeneous materials, and more specifically composites [23]. In fact, in homogeneous specimens made of poly (methyl methacrylate) (PMMA) the consideration of two-parameter models has been reported to increase by a significant amount the accuracy of the estimated results $[14,15]$, both when the T-stresses or the $A_{1 / 2}$ stresses are considered. Currently there exists no conclusive proof as to which nonsingular term should be preferred over the other in brittle materials [13, 15], albeit the need to include extra terms is clear.

The importance of the non-singular stresses in brittle materials can also be visually identified by observing the near-tip stress field by means of photoelasticity [25-29]. For homogeneous specimens, the photoelastic fringe pattern under mode I loading follows a concentric ellipsoidal pattern, originating at the crack tip. This fringe pattern can then be numerically reconstructed and compared with the experimentally obtained images in order to identify the number of terms needed to accurately approximate numerically the experimental results. In all studies, the inclusion of non-singular terms in the reconstruction of the near-tip field increased the accuracy of the estimations, while often multiple additional terms were needed to precisely capture the experimental results.

The significance of non-singular stresses on the size effect observed in porous materials has been previously investigated by the authors numerically [30], where a two-dimensional, quasi-isotropic material with circular pores arranged in an equilateral triangular array was studied in mode I fracture. The results showed that the existence of porosity can significantly reduce the size of the K-dominant zone, causing variations in the estimated stress distribution at the crack tip for different specimen sizes and porosity levels.

In the present study, mode I experiments were performed on double cantilever beam (DCB) specimens, perforated with arrays of holes. The stress at the crack tip for a given load was estimated to confirm the existence of a size effect in brittle porous materials, while the specimens were observed by means of photoelasticity to provide proof of the influence of the pores on the near-tip stress field. A numerical model was then created and the results produced were compared against the experimental results to investigate the 
influence of non-singular stresses on the specimens' behaviour. A parametric investigation was subsequently carried out to investigate the impact of manufacturing inaccuracies in the pores' shape on the measured properties.

\section{Methodology}

\subsection{Experimental methods}

There currently exists no experimental testing standard for mode I fracture of brittle porous materials. The most commonly used testing standard found in the literature is ASTM D5045 (e.g.[5-7]), which refers to compact tension (CT) and single-end-notch bending (SENB) specimens of non-porous plastic materials. In this study, the linear elastic progression towards fracture is studied, rather than the fracture toughness value which is usually the value of interest. Thus, precise measurements are required during the specimens' loading. Consequently, double cantilever beam (DCB) specimens were preferred which can achieve larger displacements at the loaded end for smaller loads. Thus, specimen design and testing was mainly based on ASTM D5528, while ASTM D5045 was consulted for insights on polymer material testing specifics.

The specimens were manufactured by laser-cutting a $10 \mathrm{~mm}$ thick PMMA sheet, procured by Stockline plastics. The selected sheet thickness was used to ensure plane strain throughout the specimen. PMMA was selected as it is both brittle and transparent, allowing for the visualisation of the near-tip stress field using photoelasticity. Cast PMMA was preferred over extruded sheets to ensure a greater degree of isotropy in the specimens' behaviour. The laser-cutting was done on the Speedy 400 machine by Trotec, using a program job control with the following parameters: Power $=100 \mathrm{Watts}$, Speed $=0.1 \mathrm{inch} / \mathrm{sec}$, Frequency $=5000 \mathrm{hz}$. The lens used was a Silicon REFLECTOR with a $24.4 \mathrm{~mm}$ diameter and a thickness of $6 \mathrm{~mm}$. However, since the results presented here are normalised with the value of the homogeneous case and the materials studied are brittle, it can be argued that the normalised values and relative differences between specimens of different configurations would remain the same, regardless of the specific manufacturing conditions or material properties.

In order to address the size effect, specimens of three different sizes were examined, each one being four times larger than its smaller counterpart (specimen of 1,2 and 4 rows of voids per arm, $\mathrm{Nc}_{\mathrm{y}}$, were manufactured). The aspect ratio of the specimens was kept constant at $W / h=12$ for all sizes, 


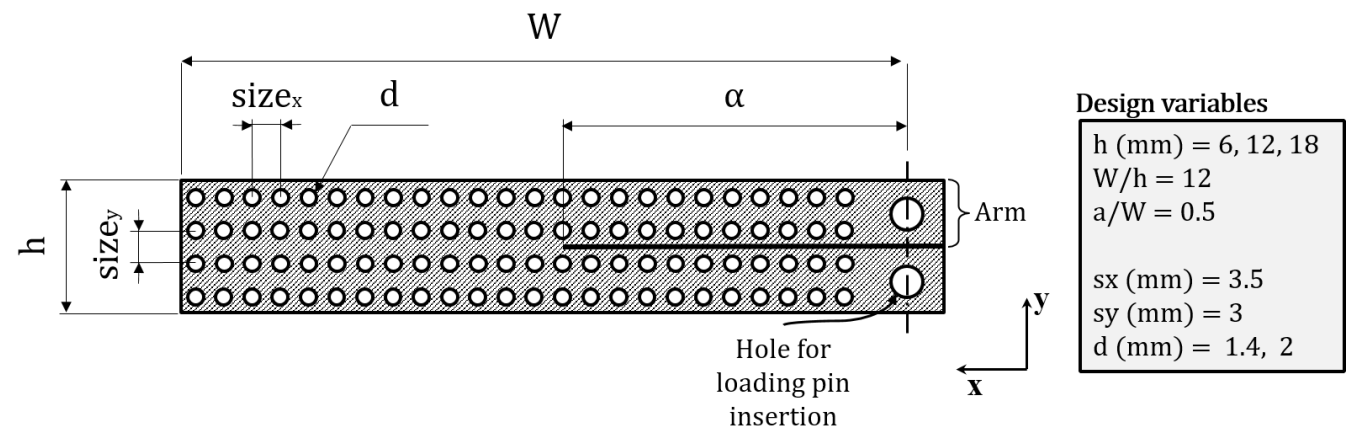

(a)

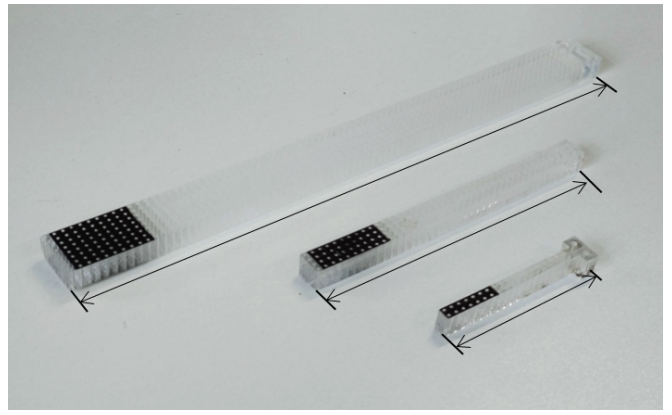

(b)

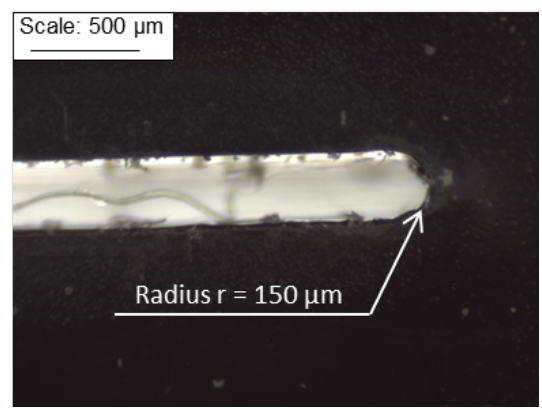

(c)

Figure 1: Designed and manufactured specimens. (a) Design variables. The definition of the axes $x$ and $y$ are shown on the figure, with $x$ being in the direction of the crack propagation. The same orientation of all pictures of the specimens is kept throughout the chapter.

(b) Manufactured specimens of all three different sizes. (c) Crack tip as seen in an optical microscope. The crack tip radius was found to be $150 \mu \mathrm{m}$.

where $\mathrm{W}$ and $\mathrm{h}$ are the specimen's length and height respectively as depicted in Figure 1a. Using laser-cutting, a horizontal crack was created in the midplane of each specimen, with a crack length to total specimen length ratio approaching $\alpha / W=0.5$, where $\alpha$ is the crack length. It has been shown [30] that the local stress intensity oscillates greatly for varying locations of the crack tip with respect to the nearest void. In order to produce comparable results, in all cases the crack tip was designed to be directly below the void, thus producing small variations in the value of the $\alpha / W$ ratio.

Note that the crack was laser cut and was not sharpened further with the use of a sharp razor, as described in ASTM D5045. Due to the different specimen configuration (here, DCB is used instead of SENB or CT, which 
are suggested in ASTM D5045), the insertion of a razor in the crack tip is a lot more challenging and would require a very thin razor. However, when a razor of $100 \mu \mathrm{m}$ was used, it was not stiff enough to further sharpen the crack tip. Figure 1c shows the near-tip area of the manufactured specimens with the laser-cut crack, magnified using an optical microscope. The crack-tip radius was measured to be $150 \mu \mathrm{m}$ (Figure 1c). Previous studies have shown that the pre-conditioning of the crack tip can impact the measured fracture toughness [31, 32] and fracture mechanics dictates that the stress intensity would be lower for blunt notches. However, in this study the focus is on the relative stress intensity differences between specimens of different sizes and porosity levels. As such, all values obtained from the porous specimens will be normalised by the values of the respective homogeneous specimens, which are manufactured by the same way and are characterised by the same crack tip radius. Thus, it is expected that this crack tip imprecision will be normalised out.

Porosity in the specimens was introduced in the form of circular voids, arranged on a square array. Two different levels of porosity were examined, namely 15 and $30 \%$, that correspond to a void diameter of approximately 1.4 and $2 \mathrm{~mm}$ respectively. The distance between consecutive voids was designed as $S i z e_{\mathrm{x}}=3.5 \mathrm{~mm}$ and $S i z e_{\mathrm{y}}=3 \mathrm{~mm}$ in the $\mathrm{x}$ - and y-direction respectively (see Figure 1a). A series of homogeneous specimens was also manufactured and tested in order to provide a baseline for normalisation. Three specimens were tested for each different configuration, leading to a total of 27 specimens (3 specimens, 3 values of $N c_{\mathrm{y}}=1,2,4$ and 3 porosities $P=0 \%, 15 \%$ and $30 \%)$.

Before testing, the manufactured specimen (Figure 1b) were subjected to a quality control procedure to ensure their geometrical consistency. Selfsimilarity between all different types of specimens was validated by means of weighing each specimen. In order to assess the fidelity of the manufactured specimens to the design specifications, each specimen's surface was scanned and the image was then processed in an in-house MATLAB code to extract all values relevant to the designed void pattern, including the void's dimensions and inter-void distances.

Mode I testing was conducted on an Instron Electroplus E3000 with a constant loading rate of $5 \mathrm{~mm} / \mathrm{min}$ to avoid viscoelastic deformations. The specimens were loaded up to a certain value, below the critical point, and then unloaded at a constant rate. Note that the specimen remained within the linear elastic region and crack propagation was not reached. ASTM D5528 
suggests a number of correction methods to account for the effects caused by crack front rotation on the estimated energy release rate $G_{I}$. However, in this study, since the specimens did not reach crack propagation, these methods are not applicable and thus $G_{I}$ was estimated from the load-displacement graphs using the simple beam theory expression for the DCB specimens:

$$
G_{I}=\frac{3 F \delta}{2 B \alpha}
$$

, where $\mathrm{F}$ here is the load and $\delta$ is the displacement.

This study though, is focused on the stress intensity on the crack tip, instead of the energy release rate. Since the material is assumed to remain in the linear elastic region, and plane strain conditions prevail, the stress intensity $K_{I}$ (not to be confused with the critical stress intensity factor) was then estimated from the energy release rate $G_{I}$ as:

$$
K_{I}=\sqrt{\frac{G_{I} E}{1-v^{2}}}
$$

During loading, the near-tip stress field was monitored with the use of a linear polariscope to obtain qualitative results on the influence of porosity on the near-tip stress field.

\subsection{Numerical approach}

In order to validate the experimental results, two-dimensional Finite Element (FE) models of the specimens were created, using the commercial software ANSYS. The cornerstone of the model is a rectangular unit cell of size $S i z e_{\mathrm{x}}=3.5 \mathrm{~mm}$ and $S i z e_{\mathrm{y}}=\sqrt{3} S i z e_{\mathrm{x}} / 2 \approx 3 \mathrm{~mm}$ in the $\mathrm{x}-$ and $\mathrm{y}$ - direction respectively (Figure 2). Each unit cell contains a single void whose centre is coincident with the centre of the unit cell.

The 8-node two-dimensional element Plane 183 was used to create a structured mesh on each unit cell, comprising of 16 and 14 equal-sized elements in the vertical and horizontal direction respectively. In order to achieve mesh convergence in the near-tip area, the horizontal boundaries of the unit cells immediately adjacent to the crack-tip are refined $4 \mathrm{x}$ times, giving a total of 56 and 16 elements in the horizontal and vertical direction respectively.

By repeatedly regenerating the described unit cell, models of different sizes were created (with a number of rows of voids $\mathrm{Nc}_{\mathrm{y}}=1,2,4$ to reflect the manufactured specimens), with a constant aspect ratio of 12 for all cases. Due to the symmetric nature of the mode I tests, only half the model was 
generated and appropriate symmetry boundary conditions were added on the model.

As in the experimental case, porosity was introduced in the form of circular voids, placed on a square array with diameters equal to 1.4 and $2 \mathrm{~mm}$. Five different locations relative to the nearest void were considered for the crack tip position, starting at the lower right corner of the unit cell $(s x=0)$ and advancing the crack tip by one quarter of the unit cell's length up to the other end of the unit cell $(s x=1)$ (Figure 2). Note that the crack tip locations are at the bottom of the unit cell (red triangles) and voids do not intersect the crack surface. The models were then loaded with a single point load at the cracked end of the specimen. The load for each size and crack length advancement $s x$ was scaled, so that a constant moment is applied on the crack tip, thus mainting the stress intensity of all homogeneous specimens at a constant value. Since the estimated values of the stress intensity are all normalised with the respective values of the homogeneous case, and given the fact that the behaviour of the studied specimens is linear elastic, the exact value of the point load is inconsequential on the values presented below. In this study, a perfectly brittle material was assumed with a matrix Young's modulus of $E=3 G P a$ and a Poisson ratio $v=0.35$. Yet, again, the specific values used are insignificant, as all results will be normalised.

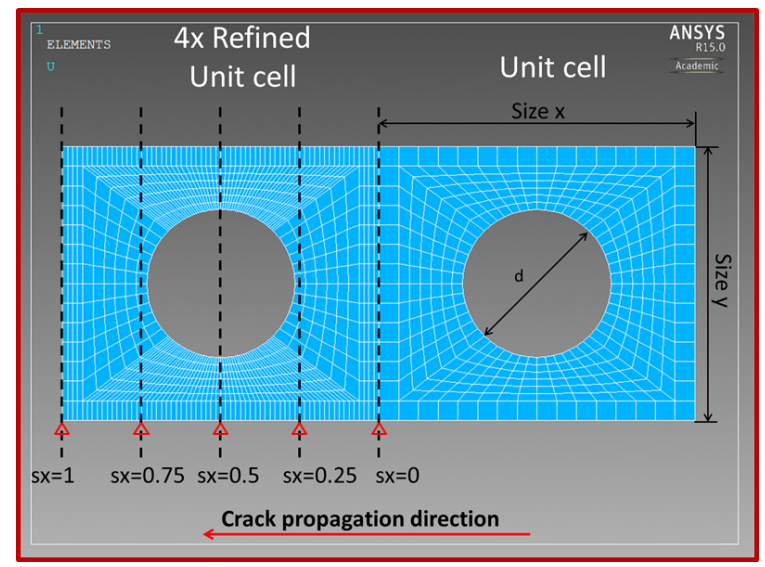

Figure 2: Unit cells used for the numerical models. The unit cell on the left is $4 \mathrm{x}$ refined compared to that on the right and was used near the crack-tip to improve numerical accuracy. The crack tip locations with respect to the void, $s x$, are also pictured here. The crack was always assumed to be facing towards the direction of the arrow. The red triangles at the bottom of the unit cell represent the crack tip locations. 
For each model the locations of all nodes in the extension of the crack tip were exported, along with the y-direction nodal stresses at each node. The stress intensity and amplitude of non-singular stresses at each location was then estimated by using the projection method (previously used by the authors in [30], originally presented in [33]), which is briefly described below.

The in-plane near-tip stress field can be described by the William's asymptotic expansion [17] as:

$$
\begin{aligned}
& {\left[\begin{array}{c}
\sigma_{\mathrm{rr}} \\
\sigma_{\theta \theta} \\
\tau_{\mathrm{r} \theta}
\end{array}\right]=} \frac{K_{\mathrm{I}}}{\sqrt{2 \pi x}} \frac{1}{4}\left[\begin{array}{c}
-\cos \frac{3 \theta}{2}+5 \cos \frac{\theta}{2} \\
\cos \frac{3 \theta}{2}+3 \cos \frac{\theta}{2} \\
\sin \frac{\theta}{2}+\sin \frac{3 \theta}{2}
\end{array}\right]+4 A_{0}\left[\begin{array}{c}
\cos ^{2} \theta \\
\sin ^{2} \theta \\
-\sin \theta \cos \theta
\end{array}\right] \\
&+\frac{3 A_{1 / 2} r^{1 / 2}}{4}\left[\begin{array}{c}
-\cos \frac{3 \theta}{2}+5 \cos \frac{\theta}{2} \\
\cos \frac{3 \theta}{2}+3 \cos \frac{\theta}{2} \\
\sin \frac{\theta}{2}+\sin \frac{3 \theta}{2}
\end{array}\right]+\begin{array}{l}
\text { Higher } \\
\text { Order } \\
\text { Terms }
\end{array}
\end{aligned}
$$

When considering only the first three terms, the stresses in the y-direction ahead of the crack tip $(\theta=0)$, where $\mathrm{x}$ can be substituted for $\mathrm{r}$, is given by:

$$
\sigma_{\mathrm{yy}}(x)=\frac{K_{\mathrm{I}}}{\sqrt{2 \pi x}}+3 A_{1 / 2} \sqrt{x} \Longrightarrow \sigma_{\mathrm{yy}}(x) \sqrt{2 \pi x}=K_{\mathrm{I}}+\left(3 \sqrt{2 \pi} A_{1 / 2}\right) x
$$

When plotting Equation (4), the value $\left(3 \sqrt{2 \pi} A_{1 / 2}\right)$, which constitutes the amplitude of the $\mathrm{A}_{1 / 2}$ non-singular stresses, is represented by the slope of the linear part of the stress distribution as $\mathrm{x}$ approaches zero. In this study, this value is then normalised for size by multiplying with the crack length $\alpha$ and is averaged for sx $=0-0.75$, in order to produce a single value for each porosity level and specimen size. This is referred to as the averaged normalised amplitude of $\mathrm{A}_{1 / 2}$-stresses $\tilde{C}=\left(3 \sqrt{2 \pi} A_{1 / 2}\right) \alpha$.

For infinitesimal distances from the crack-tip, Equation (4) converges to the value of the stress intensity, which in this study is denoted with the symbol, $S I$, in order to differentiate it from the critical stress intensity or fracture toughness. This value is then normalised by dividing it by the stress intensity for the homogeneous case, $S I_{\mathrm{H}}=31.81 M P a \sqrt{m m}$. The estimated stress intensity factors for all different crack tip locations sx $=0-0.75$ of each type of model were then averaged to obtain a topology independent value, which is referred to as the averaged normalised stress intensity, $\bar{S} I_{\mathrm{N}}$.

The size of the K-dominant zone is estimated as the size of the region inside which the analytical singular field can describe the stress-field to at 
least a 95\% degree of accuracy. This degree of K-dominance is estimated as:

$$
\Lambda=\frac{\sigma_{\mathrm{y}}{ }^{\operatorname{sing}}}{\sigma_{\mathrm{y}}{ }^{\operatorname{sing}}+\left|\sigma_{\mathrm{y}}{ }^{\text {non-sing }}\right|}
$$

, where

$$
\sigma_{\mathrm{y}}{ }^{\operatorname{sing}}(x)=\frac{K_{\mathrm{I}}}{\sqrt{2 \pi x}} \quad \text { and } \quad \sigma_{\mathrm{y}}{ }^{\text {non-sing }}(x)=\sigma_{\mathrm{yy}}(x)-\sigma_{\mathrm{y}}{ }^{\operatorname{sing}}(x)
$$

\section{Results}

The isochromatic fringes for all cases are presented in Figure 3, along with the ones predicted from FEA. Qualitatively, the experimental results appear to generally agree with the numerically computed results in all different cases. This acts as a preliminary validation of the numerical results.

For the homogeneous case (see Figures 3c 3d, 3g and 3h) we can see, both experimentally and numerically, that the isochromatics follow a smooth pattern, with ellipsoidal hoops expanding behind the crack-tip for a certain length, as is expected for Mode I loading [27, 29]. The photoelastic fringe for all sizes of the homogeneous case exhibit the same trends in shape, with the zone in which these stresses appear seeming to scale proportionally with size.

The isochromatics presented in porous materials show a quite different picture (Figures 3a, 3b, 3e and 3f). The perforations in the material seem to act as a disturbance in the expansion of the stress field in the near-tip area, altering the general shape and size of the stress distribution. In fact, for specimens with just one row of unit cells per arm (Figures 3a, 3b), the isochromatic fringe is mildly changed through the existence of the voids, though retaining its similarities to the homogeneous case. However, in larger specimens (Figures 3e, 3f) a more chaotic pattern is exhibited, with seemingly little correlation to the original, ellipsoidal hoops. The FEA models capture the size and general shape of these stress fields satisfactorily.

The photoelastic fringe pattern has been used in previous research to experimentally estimate the stress intensity factor and indicate the need of the inclusion of non-singular terms in the estimation of the stress intensity factor in homogeneous specimens of varying sizes and configurations [25-28]. In porous materials, due to the widely irregular nature of the fringe pattern, 


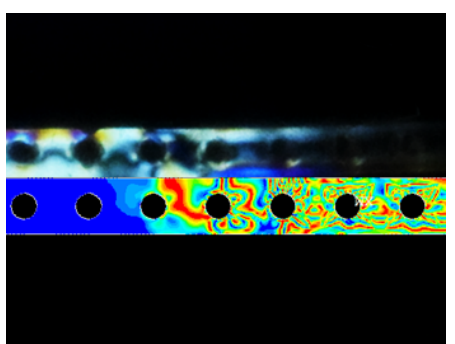

(a)

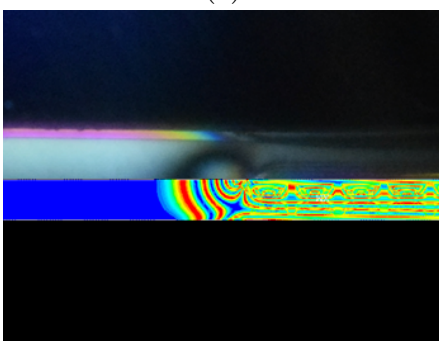

(c)

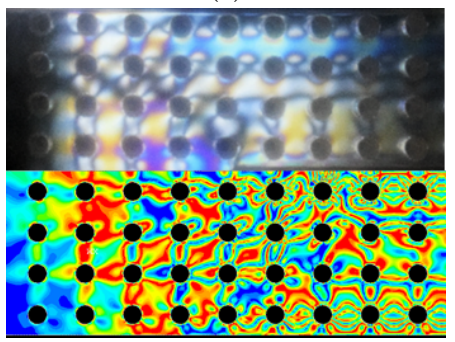

(e)

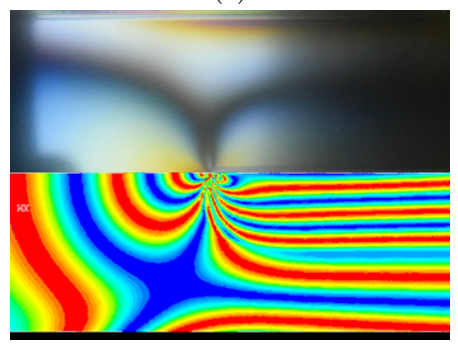

(g)

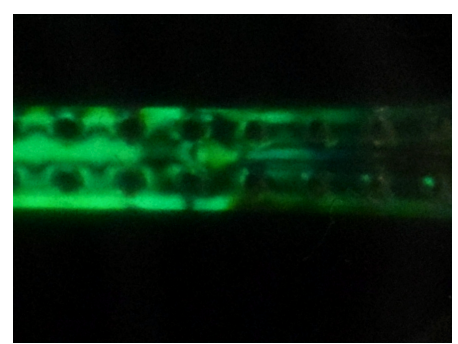

(b)

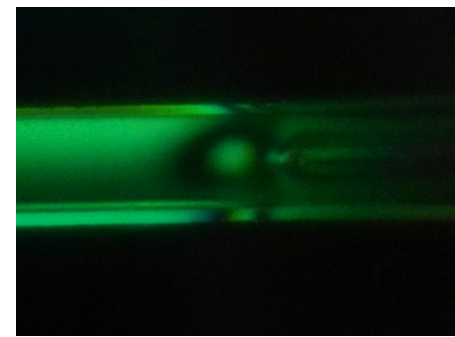

(d)

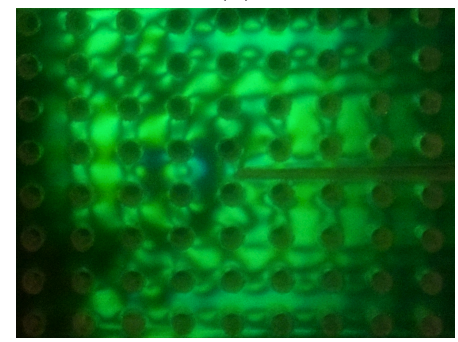

(f)

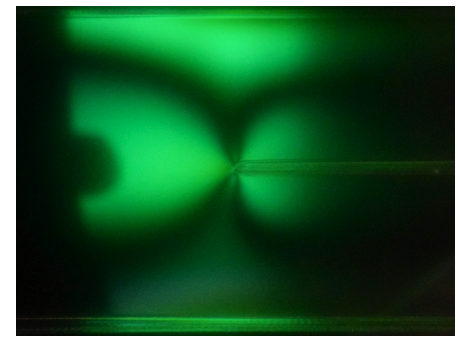

(h)

Figure 3: Isochromatic fringes in specimens during mode I loading. Left side: Fringe patterns (top) and respective FEA results (bottom). Right side: Dark field isochromatics. (a) \& (b): Porous specimen with $\mathrm{Nc}_{\mathrm{y}}=1$, (c) \& (d): Homogeneous specimen with $\mathrm{Nc}_{\mathrm{y}}=1$, (e) \& (f): Porous specimen with $\mathrm{Nc}_{\mathrm{y}}=4$, (g) \& (h): Homogeneous specimen with $\mathrm{Nc}_{\mathrm{y}}=4$ 
the coefficients of the Williams series cannot be extracted. However, a visual inspection clearly shows that the existence of pores acts as a disturbance in the expansion of the stress field in the near-tip area, which could justify the need to include non-singular terms, in addition to the singular term, to accurately reconstruct the isochromatics pattern.

Quantitatively, testing revealed consistent behaviour between all specimens of different types, with minimal standard deviations (Figure 4), ensuring the repeatability of the presented results. It is important to note that the experimental results only refer to one location of the crack tip with respect to the void. As described in the methodology section, the specimens
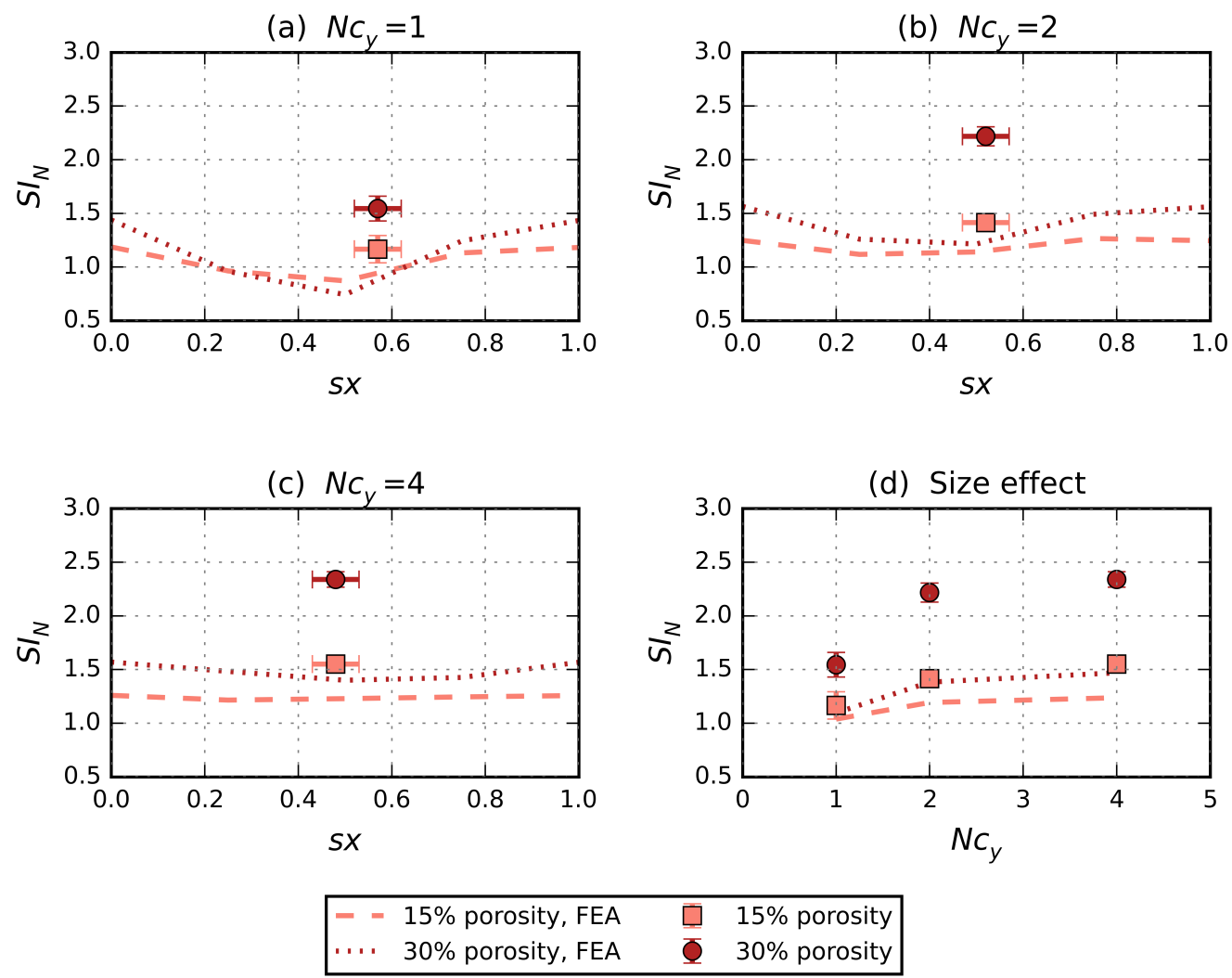

Figure 4: Numerical variation in $\mathrm{SI}_{\mathrm{N}}$ within unit cell with experimental data points appended for each different size: (a) $\mathrm{Nc}_{\mathrm{y}}=1$, (b) $\mathrm{Nc}_{\mathrm{y}}=2$, (c) $\mathrm{Nc}_{\mathrm{y}}=4$. (d)Experimental size effect with numerical results averaged for sx $\left(\bar{S} I_{N}\right)$. Even though the numerical predictions underestimate the experimental results, they both exhibit the same trends. 
were designed so that the crack tip location with respect to the voids is located below their centre (or $\mathrm{sx}=0.5$ as seen in Figure 2 ). However, due to manufacturing constraints, the total crack length had a variation of $0.5 \mathrm{~mm}$, which, given the small size of each unit cell, results in crack tip locations in the range of approximately $\mathrm{sx}=0.4-0.65$.

Figures $4 \mathrm{a}, \mathrm{b}, \mathrm{c}$ show the numerically predicted variation of the normalised stress intensity within a unit cell, plotted along with the results of the experiments. The experimental data points are placed in the $\mathrm{x}$ axis at the approximate location where the crack tip was found to be located from the quality control procedure for each specimen size. For all different sizes and porosities, the numerical results have underestimated the experimental values. In fact, the higher the porosity, the larger the deviation between the experimental and numerical results.

These deviations can be attributed to limitations during manufacturing. During the quality control of the specimen it was found that even though the topology of the voids was accurately manufactured, the voids' diameters exceeded the designed value by a factor of approximately $10 \%$ (i.e. $0.14 \mathrm{~mm}$ ) which is attributed to laser stretching. Additionally, the circularity of the voids was found to deviate by an averaged factor of 0.15 , while the average eccentricity of the voids was estimated to be 0.35 . In the specimen with the $2 \mathrm{~mm}$ void diameter, due to the close tolerances, the crack was sometimes found to be located slightly off from the mid-plane, which could give rise to mode II phenomena as well. Nevertheless, this study aims to highlight trends in the behaviour of porous materials in mode I fracture and not validate precise numerical values.

With the exception of the specimen with $\mathrm{Nc}_{\mathrm{y}}=1$, the numerically predicted variation of the stress intensity around the void is limited (Figures $4 \mathrm{a}, \mathrm{b}, \mathrm{c})$. At the same time, the crack tip for each manufactured specimen type might have minimal variations in its crack tip location sx, as described previously. Therefore, an averaged value of the numerical results for $\mathrm{sx}=$ $0-0.75$ ( $\mathrm{sx}=1$ is excluded due to periodicity) is used as a baseline for the experimental results. The experimental results are compared to the averaged baseline in Figure 4d.

As expected, porous materials appear weaker than the homogeneous material $\left(S I_{N}=1\right.$ for all $s x$ values of the homogeneous case), with the stress intensity at the crack tip increasing for increasing porosity. Experimental findings also show a strong size effect, with smaller specimen exhibiting lower normalised stress concentration at the crack tip than their larger counter- 
parts, which are then seemingly converging to a constant value for increasing size. This behaviour is consistent with the numerically predicted results for both, the exact representation of the tested specimens, and specimens of similar configuration, where the voids were placed in a triangular array and the specimen's aspect ratio was large enough to assume the existence of a long crack [30].

Previously, the authors have correlated this size effect to the significance of the $\mathrm{A}_{1 / 2}$ non-singular stresses [30]. It was found that even $5 \%$ porosity can shrink the size of the K-dominance zone to $50 \%$ compared to the homogeneous case. Thus, it was suggested that in the case of porous materials it cannot be safely assumed that the non-singular stresses can be neglected. In fact, the normalised stress intensity at the crack tip was found to be empirically correlated by a geometrical parameter $\left(F_{1}=\sqrt{P / N c_{\mathrm{y}}}\right)$ with the $\mathrm{A}_{1 / 2^{-}}$ stresses, which in turn can be uniquely described by a function of a different geometrical parameter $\left(F_{2}=1 / N c_{\mathrm{y}} P\right)$. Both parameters were functions of the specimen's porosity, $P$, and number of rows of voids in the vertical direction, $\mathrm{Nc}_{\mathrm{y}}$, and by dividing $F_{2}$ with $F_{1}$ we get the geometrical dependency coefficient:

$$
D_{\mathrm{c}}=\sqrt{\frac{1}{N c_{\mathrm{y}} P^{3}}}
$$

Based on this coefficient, for the idealised models studied previously by the authors [30] (where the voids triangular topology implied quasi-isotropic behaviour and the specimen's aspect ratio ensured a long crack), a piecewise model was empirically derived, with a unique function for models with just one row of unit cells per specimen arm (black dashed line on Figure 5, $\bar{S} I_{\left.N\right|_{N c_{y}=1}}$ ) and a different, unique function for larger models (black dotted line on Figure $5, \bar{S} I_{\left.N\right|_{N c_{y}=2-6}}$ ). The data used for this model are the averaged values of the numerically predicted $S I_{N}$, for values of $s x=0-0.75$ of the idealised models.

The empirical model of [30] largely underestimates the values of the experimental results found in this study. As the idealised numerical model and the numerical model representing the experimental results are not expected to deviate much (which can also be seen by comparing the values of the averaged $S I_{N}$ between the two papers), the deviation of the empirical model with the epxerimental results is attributed to the manufacturing inaccuracies of the specimens, as also described above. 


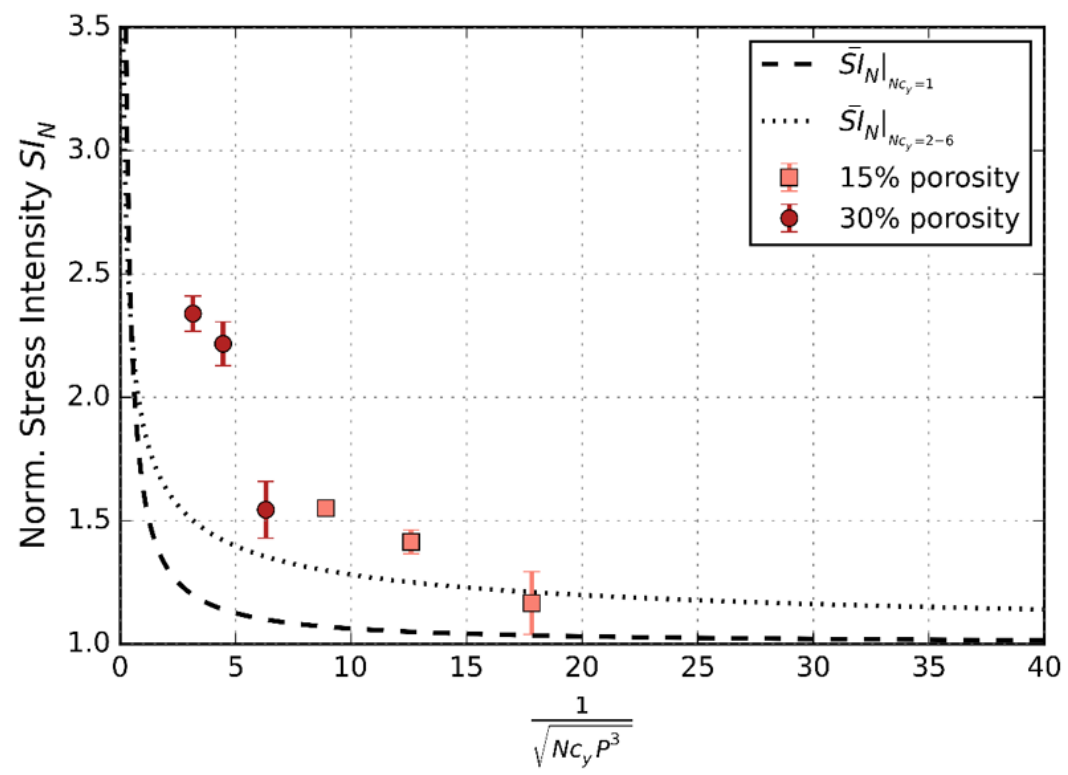

Figure 5: Comparison of experimental results with fitted model from [30]. The numerical model for $\left.S I_{\mathrm{N}}\right|_{\mathrm{Nc}_{\mathrm{y}=1}}$ represents specimens with one row of per voids per specimen arm, while the one labelled $\left.S I_{\mathrm{N}}\right|_{\mathrm{Nc}_{\mathrm{y}}=2-6}$ represents larger specimens.

Still, the experimental results of this study appear to be strongly correlated with the parameter $D_{\mathrm{c}}$ (Figure 5) and the general trends indicated by the empirical models remain, with specimens of lower $D_{c}$ values, exhibiting a sharp increase in their stress intensity. Additionally, specimens with $N c_{y}=1$ exhibit lower stress intensity values, that appear to comply with numerical results, which also predict a distinct curve for models with just one row of unit cells per specimen arm. Hence, the experimental results provide further evidence that the suggested geometrical coefficient $D_{\mathrm{c}}$ is linked with the stress intensity at the crack tip of a porous material.

\section{Effects of manufacturing inaccuracies on the stress intensity at the crack tip}

The above experimental study validates the size effect and further supports the significance of non-singular stresses in specimen with arrays of voids. However, during manufacturing of such structures, the voids deviate from the ideally designed shape and can have different eccentricity or 
circularity values. In fact, porous materials occurring from more stochastic procedures than a pre-designed laser cutting (e.g. water blown free rise polyurethane foams) can have highly anisotropic void shapes. The ellipsoidal shape of the voids formed during foaming has been found to greatly influence the material's stiffness during various loading types like tensile [34, 35], compression [36, 37], and impact loading [38]. With respect to mode I fracture, the influence of void anisotropy has been investigated for ductile materials [39] and cases where the crack tip is located in the void, for infinite materials [40]. To the authors knowledge, the effect of the shape of a void in the near tip area on the stress intensity and the size of the K-dominance zone in brittle, finite size specimens, has not yet been addressed.

In order to investigate the impact of the voids' shape on the stress concentration at the crack tip, a parametric study was conducted. Models with elliptic voids (to account for differences in eccentricity) and square voids with rounded edges (to account for differences in circularity) were considered (Figure 6). The elliptic voids have a closer resemblance to the natural cell shape that is formed in free-rise foams as the gas trapped in the liquid foam rises to the surface before it solidifies. The shape anisotropy ratio, S, of the elliptic voids is considered as the ratio of the void size in the $\mathrm{x}$ direction over the void size in the y direction. For this study two different shape anisotropy ratios were considered, one where the $\mathrm{x}$-direction void size is double that of the $y$-direction $(S=2$, Figure $6 a)$ and one where it is half of it $(S=0.5$, Figure $6 \mathrm{~b})$. Cases more exaggerated than those that occurred from manufacturing inaccuracies were considered to highlight the differences.

For most cases, models of three porosities were created, approximating 5, 20 and 30\%. Note that for the case of the elliptic voids elongated in

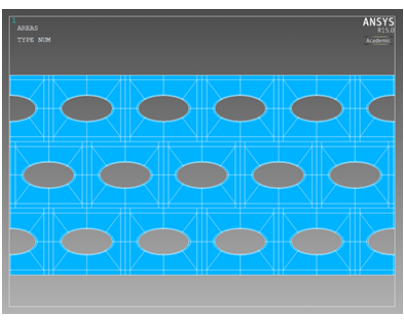

(a)

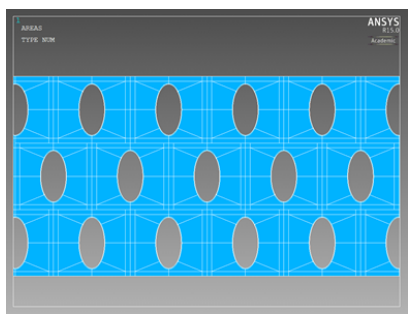

(b)

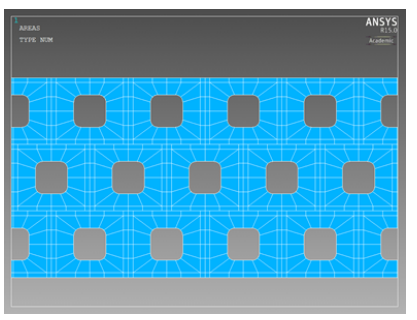

(c)

Figure 6: Models with different void shapes investigated in parametric analysis. (a) Voids elongated in x-direction, $O V, S=2$, (b) voids elongated in y-direction, $O V, S=0.5$, (c) voids with rounded rectangle shape, $S Q$ 
the y-direction $(S=0.5)$, the void diameter corresponding to $30 \%$ porosity intersected the unit cell's horizontal boundaries and thus was not considered. During this parametric study, the voids in all models are distributed in an equilateral triangle topology, to approximate isotropy.

Figure 7a plots the normalised stress intensity of all models against their size. Results show that the orientation of the elliptic voids significantly changes the material's behaviour. More specifically, when the voids major axis is parallel to the crack, the stress intensity at the crack tip diminishes, tending towards the behaviour of the homogeneous specimens and implying apparently tougher behaviour than their perfectly circular equivalent. On the contrary, when the void is rotated $90^{\circ}$ so that the major axis of the void is perpendicular to the direction of the crack, the stress intensity at the crack tip rapidly increases. Models with rounded rectangle voids exhibited a very similar behaviour to the idealised case of circular voids, although for higher porosities the stress intensity at the crack tip was slightly diminished. Despite the large differences exhibited by the stress intensity, the size of the K-dominant zone remains almost constant for all cases (Figure 7b) and any variations in it are not enough to justify the large differences in the stress intensity estimation. Thus, non-singular effects are not uniquely responsible for changes in the observed stress intensity at the crack tip.

Figure 8 a plots the amplitude of the $\mathrm{A}_{1 / 2}$-stresses, $\tilde{C}$, against the geometrical parameter $F_{2}$, which was shown in [30] to uniquely describe the variation of $\mathrm{A}_{1 / 2}$-stresses with the material's geometrical features. Indeed, even for the case of porous materials with different void shapes, the amplitude of $A_{1 / 2}$-stresses seems to agree with the value of the idealised case of circular voids, represented in Figure $8 \mathrm{a}$ by the black dotted lines. Different dotted lines represent different specimen sizes, though their exact labelling is not important in this case.

Conversely, when plotting the normalised stress intensity $\mathrm{SI}_{\mathrm{N}}$ against the geometrical parameter $F_{1}$, multiplied by the normalised amplitude of $\mathrm{A}_{1 / 2^{-}}$ stresses (Figure 8b), the parametric models deviate from the idealised case of circular voids (again, the black, dotted lines on the graph), forming distinct curves for each different void shape. Yet, the trends between these two plotted values remains the same, even if there is not a unique correlation for all material topologies.

This deviation from the idealised case is also reflected when plotting the results as a function of the geometrical parameter $\mathrm{D}_{\mathrm{c}}$ (Figure 9). Even though the previously estimated empirical model (dotted lines) can provide an ap- 
(a)

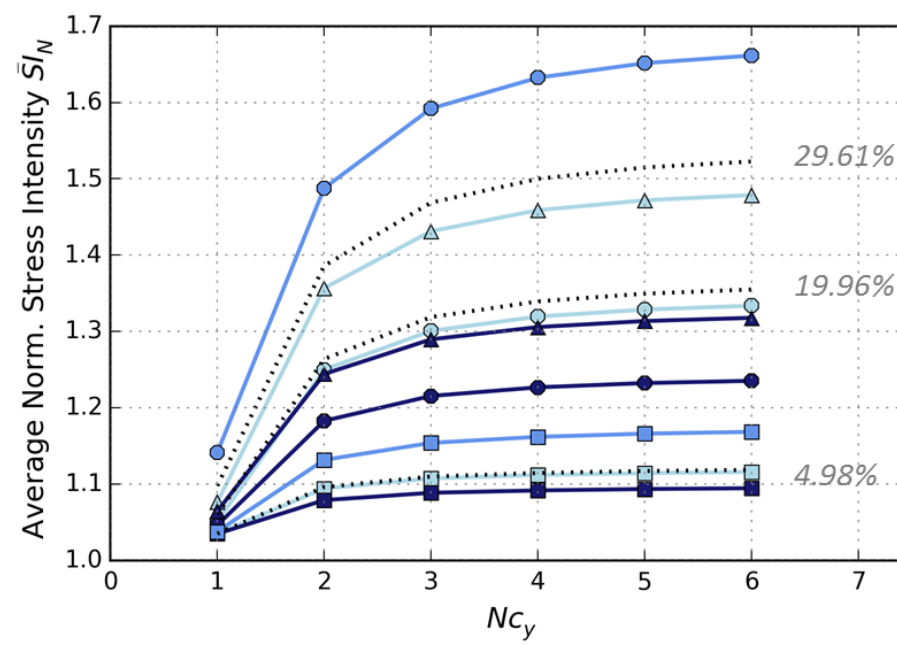

\begin{tabular}{|ll|}
\hline & Colours \\
- & $O V, S=2$ \\
0 & $O V, S=0.5$ \\
0 & $S Q$ \\
& \\
& Markers \\
- & $P=4.98 \%$ \\
- & $P=19.96 \%$ \\
. & $P=29.61 \%$ \\
$\ldots .$. & Idealised [30] \\
\hline
\end{tabular}

(b)

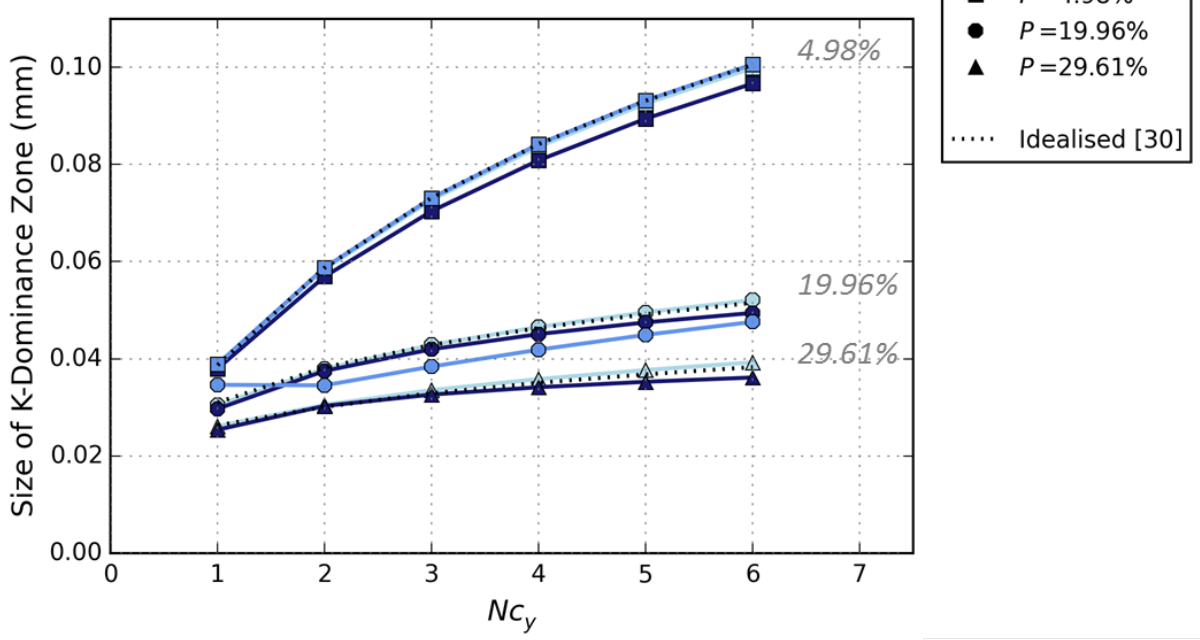

Figure 7: Influence of void shape on size effect of porous materials. Dotted lines represent the idealised case of a perfectly circular void. (a) Normalised stress intensity at the crack tip for different void shapes, (b) Size of K-dominance zone versus the size of the specimen. The annotated percentages on both graphs refer to the idealised case.

proximate, average estimation of the expected stress intensity for all different models, it is not an accurate representation. Nevertheless, each model of a different void shape follows a similar pattern, despite the difference in their exact values. This provides further evidence that the geometrical dependency coefficient $\mathrm{D}_{\mathrm{c}}$ has a strong influence on the stress intensity at the crack tip of porous materials. The experimental results are also plotted on Figure 9 
for comparison.

The presented results show that during mode I fracture, the void shape of porous materials significantly affects the stress at the crack-tip. While nonsingular stresses, $\mathrm{A}_{1 / 2}$, still appear to have a direct impact on the scaling of the normalised stress intensity for different sizes and porosity levels, the

(a)
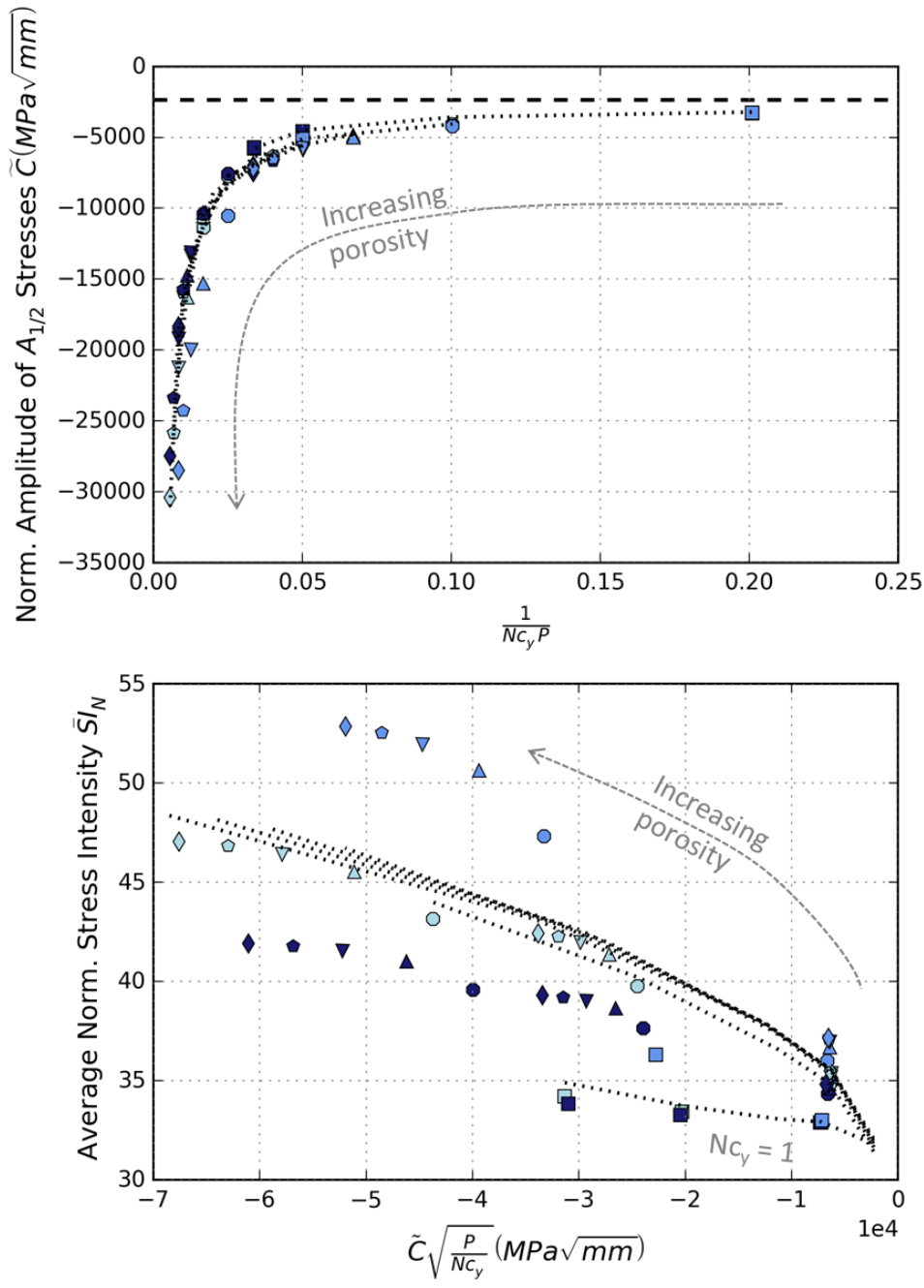

\begin{tabular}{|ll|}
\hline Colours & OV,S=2 \\
- & $O V, S=0.5$ \\
& $S Q$ \\
& Markers \\
- & $N c_{y}=1$ \\
- & $N c_{y}=2$ \\
- & $N c_{y}=3$ \\
V & $N c_{y}=4$ \\
- & $N c_{y}=5$ \\
- & $N c_{y}=6$ \\
& \\
$\ldots . .$. & Idealised [30]
\end{tabular}

(b)

Figure 8: (a) Normalised amplitude of $\mathrm{A}_{1 / 2}$-stresses versus the geometrical factor $F_{2}$ that has been found to uniquely describe them for perfectly circular voids (dashed line represents the homogeneous case), (b) Average normalised stress intensity at the crack tip versus the amplitude of the $\mathrm{A}_{1 / 2}$-stresses, multiplied by the geometrical factor $F_{1}$ 


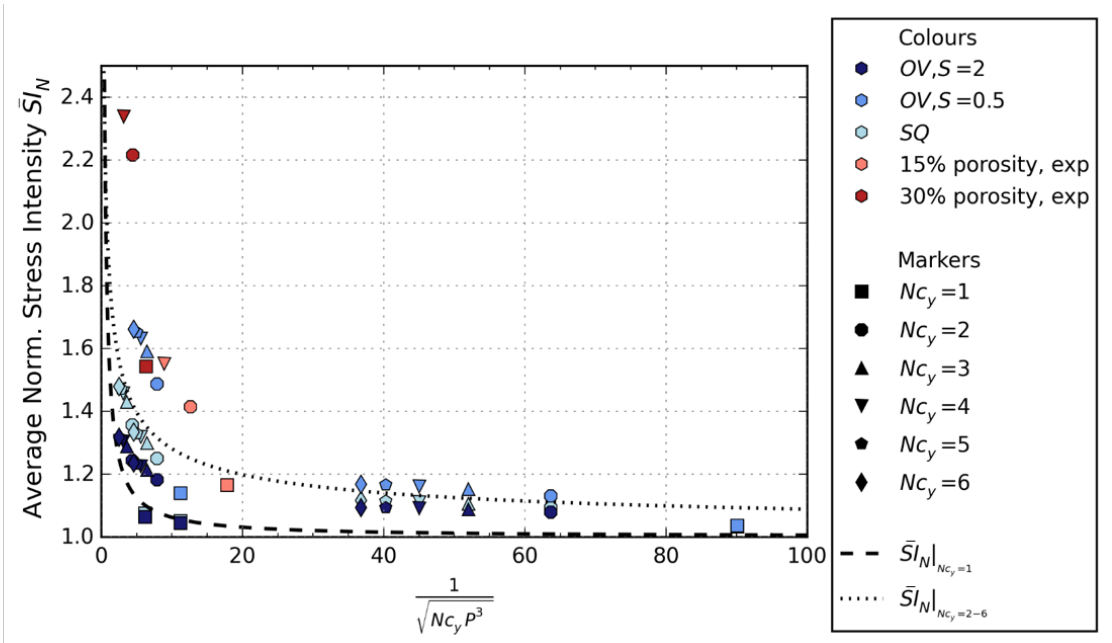

Figure 9: All parametric and experimental results against the empirical model suggested in [30]. Although exact values may differ, the general trend can be captured by the geometrical dependency coefficient $\mathrm{D}_{\mathrm{c}}$ for all cases.

exact relationship linking them together might be more complicated than the one that was previously presented for the idealised case in [30].

So far, the only geometrical parameters studied are governed by global material characteristics (the porosity and number of voids in the y direction, which is an extension of the cell density). However, the relationship between the stress intensity at the crack tip and the material's mesostructure may in fact be additionally dependent on local factors, like the proximity of the voids to the crack tip and the stochasticity of their distribution on the neartip area. An in-depth investigation should be performed, encompassing more parameters, in order to be able to achieve a better understanding of porous materials' behaviour and provide insight on how a material's mesostructure in the near-tip area can affect the material's properties.

\section{Conclusions}

The presented work investigates, both numerically and experimentally the existence of a size-effect in brittle porous materials and addresses the effect of non-singular, $A_{1 / 2}$-stresses on the material's behaviour. The key findings can be summarised as follows:

1. Mode I experiments on DCB PMMA specimens were performed to val- 
idate the existence of a size effect in porous materials. Results showed that this size effect can indeed be experimentally observed and was found to follow the same trend as the one predicted numerically. Numerical values, though, underestimated the experimental results, which can be attributed to manufacturing inaccuracies.

2. In order to address such inaccuracies, a numerical parametric analysis was conducted, investigating the influence of variations in the shape of the voids in the near-tip field. It was found that even though such variations can have a great influence on the exhibited stress at the crack tip, the amplitude of the $\mathrm{A}_{1 / 2}$ non-singular stresses remain almost unaffected for all different void shapes studied.

3. The geometrical dependency coefficient $D_{c}$ has been found to be strongly correlated with the stress at the crack tip for both experimental and numerical parametric results. However, the empirical relationship predicted in [30] does not seem to be able able to fully predict the normalised stress intensity for all cases. More parameters governing the mesostructure need to be investigated, which could also have a direct impact on material's behaviour in mode I loading, additional to the $\mathrm{A}_{1 / 2}$ non-singular stress variations.

\section{References}

\section{References}

[1] W. Weibull, The phenomenon of rupture in solids,, Generalstabens litografiska anstalts forlag, Stockholm, 1939.

URL http://www.worldcat.org/title/ phenomenon-of-rupture-in-solids/oclc/30416255

[2] Z. P. Bazant, Scaling laws in mechanics of failure (1993).

[3] Z. P. Bazant, Y. Xi, S. G. Reid, Statistical size effect in quasi-brittle structures: I. Is Weibull theory applicable?, Journal of Engineering Mechanics 117 (11) (1991) 2609-2622.

[4] Z. P. Bazant, Concrete fracture models : testing and practice, Engineering Fracture Mechanics 69 (2002) 165-205. 
[5] M. E. Kabir, M. C. Saha, S. Jeelani, Tensile and fracture behavior of polymer foams, Materials Science and Engineering A 429 (1-2) (2006) 225-235. doi:10.1016/j.msea.2006.05.133.

[6] E. Linul, L. Marsavina, T. Sadowski, M. Knec, Size Effect on Fracture Toughness of Rigid Polyurethane Foams, Solid State Phenomena 188 (2012) 205-210. doi:10.4028/www.scientific.net/SSP.188. 205. URL http://www. scopus. com/inward/record.url?eid=2-s2. 0-84861432159\&partnerID=tZOtx3y1

[7] L. Marsavina, E. Linul, Fracture toughness of polyurethane foams. Experiments versus micromechanical models., in: Proc 18th European Conference on Fracture, 2010, pp. 1-8.

[8] Z. P. Bazant, I. M. Daniel, L. Zhengzhi, Size Effect and Fracture Characteristics of Composite Laminates, Journal of Engineering Materials and Technology 118 (July) (1996) 317-324.

[9] Z. P. Bazant, Y. Zhou, D. Novák, I. M. Daniel, Size effect in fracture of sandwich structure components: Foam and laminate, American Society of Mechanical Engineers, Applied Mechanics Division, AMD 248 (2001) 19-30.

[10] J. R. Rice, Mathematical Analysis in the Mechanics of Fracture, Fracture: An Advanced Treatise Mathematical Fundamentals) N.Y 2 (1968) 191-311.

URL https://pdfs. semanticscholar.org/212d/ 0db636bcd4413aff761ed1707a5d62fe06e9.pdf

[11] T. Anderson, Fracture Mechanics: Fundamentals and Applications, 2005.

[12] J. Rice, Limitations to the small scale yielding approximation for crack tip plasticity, Journal of the Mechanics and Physics of Solids 22 (1) (1974) 17-26. doi:10.1016/0022-5096(74)90010-6.

URL http://linkinghub.elsevier.com/retrieve/pii/ 0022509674900106

[13] D. Gardeazabal, Z. He, A. Kotousov, On influence of non-singular stress states on brittle fracture, International Journal of Fracture 185 (1-2) (2014) 201-208. doi:10.1007/s10704-013-9903-7. 
[14] B. Kumar, S. Chitsiriphanit, C. T. Sun, Significance of K-dominance zone size and nonsingular stress field in brittle fracture, Engineering Fracture Mechanics 78 (9) (2011) 2042-2051. doi:10.1016/j. engfracmech. 2011.03.015.

URL http://dx.doi.org/10.1016/j.engfracmech.2011.03.015

[15] Y. J. Chao, S. Liu, B. J. Broviak, Brittle fracture: Variation of fracture toughness with constraint and crack curving under mode I conditions, Experimental Mechanics 41 (3) (2001) 232-241. doi:10.1007/ BF02323139.

[16] D. J. Smith, M. R. Ayatollahi, M. J. Pavier, On the consequences of T-stress in elastic brittle fracture, Proceedings of the Royal Society A: Mathematical, Physical and Engineering Sciences 462 (2072) (2006) 2415-2437. doi:10.1098/rspa.2005.1639.

[17] M. L. Williams, On the Stress Distribution at the Base of a Stationary Crack, Journal of Applied Mechanics 24 (3) (1957) 109-114. doi:10. 1115/1.3640470.

[18] K. Sedighiani, J. Mosayebnejad, H. Ehsasi, H. Sahraei, The effect of T-stress on the brittle fracture under mixed mode loading, Procedia Engineering 10 (2011) 774-779. doi:10.1016/J . PROENG. 2011.04.128. URL https://www.sciencedirect.com/science/article/pii/ S187770581100316X

[19] M. Rashidi Moghaddam, M. R. Ayatollahi, S. M. J. Razavi, F. Berto, Mode II brittle fracture assessment using an energy based criterion, Physical Mesomechanics 20 (2) (2017) 142-148. doi:10.1134/ S1029959917020047.

URL http://link. springer.com/10.1134/S1029959917020047

[20] M. R. Ayatollahi, J. Akbardoost, Size effects in mode II brittle fracture of rocks, Engineering Fracture Mechanics 112-113 (2013) 165-180. doi: 10.1016/j.engfracmech.2013.10.011.

URL http://dx.doi.org/10.1016/j.engfracmech.2013.10.011

[21] M. R. Ayatollahi, J. Akbardoost, F. Berto, Size effects on mixed-mode fracture behavior of polygranular graphite, Carbon 103 (2016) 394-403. doi:10.1016/j. carbon.2016.03.030. 
URL http://linkinghub.elsevier.com/retrieve/pii/ S0008622316302196

[22] M. R. Ayatollahi, M. Zakeri, An improved definition for mode I and mode II crack problemsdoi:10.1016/j .engfracmech.2017.01.027.

URL https://ac.els-cdn.com/S0013794417301303/ 1-s2.0-S0013794417301303-main.pdf?_tid= af1aa91e-aaa8-11e7-92e6-00000aab0f01\&acdnat $=1507302894$ 326236996f859f20819a442e96d6b689

[23] O. G. Kravchenko, S. G. Kravchenko, C.-T. Sun, Thickness dependence of mode I interlaminar fracture toughness in a carbon fiber thermosetting compositedoi:10.1016/j. compstruct.2016.10.088.

URL http://iranarze.ir/wp-content/uploads/2016/11/E504.pdf

[24] C. T. Sun, H. Qian, Brittle fracture beyond the stress intensity factor, Journal of Mechanics of Materials and Structures 4 (April) (2009) 743753.

URL http://msp.org/jomms/2009/4-4/p09.xhtml

[25] L. V. Stepanova, V. S. Dolgikh, V. A. Turkova, Digital photoelasticity for calculating coefficients of the Williams series expansion in plate with two collinear cracks under mixed mode loading, Information Technology and Nanotechnology.

URL http://ceur-ws.org/Vol-1904/paper35.pdf

[26] L. Stepanova, P. Roslyakov, P. Lomakov, A Photoelastic Study for Multiparametric Analysis of the Near Crack Tip Stress Field Under Mixed Mode Loading, in: Procedia Structural Integrity, Vol. 2, 2016, pp. 17971804. doi:10.1016/j.prostr.2016.06.226.

URL ww. sciencedirect.com

[27] M. R. Ayatollahi, M. Nejati, Experimental evaluation of stress field around the sharp notches using photoelasticity, Materials and Design 32 (2) (2011) 561-569. doi:10.1016/j.matdes. 2010.08.024.

URL http://dx.doi.org/10.1016/j.matdes.2010.08.024

[28] M. Guagliano, M. Sangirardi, A. Sciuccati, M. Zakeri, Multiparameter analysis of the stress field around a crack tip, Procedia Engineering 10 
(2011) 2931-2936. doi:10.1016/j.proeng. 2011.04.486.

URL http://dx.doi.org/10.1016/j.proeng. 2011.04.486

[29] K. Ramesh, S. Gupta, A. Kelkar, Evaluation of stress field parameters in fracture mechanics by photoelasticity - revisited, Engineering Fracture Mechanics 56 (1) (1997) 25-41.

[30] D. Touliatou, M. A. Wheel, K-dominance and size effect in mode I fracture of brittle materials with low to medium porosity, Engineering Fracture Mechanicsdoi:10.1016/J.ENGFRACMECH. 2018.06.041.

URL https://www.sciencedirect.com/science/article/pii/ S0013794418300432

[31] M. S. Cayard, W. L. Bradley, The effect of various pre-cracking techniques on the fracture toughness of plastics.pdf.

[32] J. M. De Souza, H. N. Yoshimura, F. M. Peres, C. G. Schön, Effect of sample pre-cracking method and notch geometry in plane strain fracture toughness tests as applied to a PMMA resin, Polymer Testing 31 (6) (2012) 834-840. doi:10.1016/j.polymertesting.2012.06.003.

[33] Y. Morioka, A simple method for determining stress intensity factors for a crack in bi-material interface, Theses and Dissertations Available from ProQuest.

URL http://docs.lib.purdue.edu/dissertations/AAI1489573

[34] M. Masmoudi, W. Kaddouri, T. Kanit, S. Madani, S. Ramtani, A. Imad, Modeling of the effect of the void shape on effective ultimate tensile strength of porous materials: Numerical homogenization versus experimental results, International Journal of Mechanical Sciences 130 (2017) 497-507. doi:10.1016/J. I JMECSCI . 2017.06.011.

URL https://www.sciencedirect.com/science/article/pii/ S002074031630710X

[35] E. Amsterdam, H. van Hoorn, J. T. M. De Hosson, P. R. Onck, The Influence of Cell Shape Anisotropy on the Tensile Behavior of Open Cell Aluminum Foam, Advanced Engineering Materials 10 (9) (2008) 877-881. doi:10.1002/adem. 200800128.

URL http://doi.wiley.com/10.1002/adem. 200800128 
[36] H. Benouali, A, L. Froyen, T. Dillard, S. Forest, F. N'Guyen, MECHANICAL BEHAVIOR OF CELLULAR SOLIDS Investigation on the influence of cell shape anisotropy on the mechanical performance of closed cell aluminium foams using micro-computed tomography, Journal of Materials Science (40) (2005) 5801-5811. doi:10.1007/s10853-005-4994-9.

URL http://matperso.mines-paristech.fr/Donnees/data03/ 396-benouali05.pdf

[37] Y. Mosleh, K. V. Bosche, B. Depreitere, J. V. Sloten, I. Verpoest, J. Ivens, Effect of polymer foam anisotropy on energy absorption during combined shear-compression loading, Journal of Cellular Plastics 54 (3) (2018) 597-613. doi:10.1177/0021955X17720156.

URL http://journals. sagepub.com/doi/pdf/10.1177/ $0021955 X 17720156$

[38] K. Vanden Bosche, Y. Mosleh, B. Depreitere, J. Vander Sloten, I. Verpoest, J. Ivens, Anisotropic polyethersulfone foam for bicycle helmet liners to reduce rotational acceleration during oblique impact, Proceedings of the Institution of Mechanical Engineers, Part H: Journal of Engineering in Medicine 231 (9) (2017) 851-861. doi:10.1177/0954411917711201.

URL http://journals.sagepub.com/doi/10.1177/ 0954411917711201

[39] X. Gao, T. Wang, J. Kim, On ductile fracture initiation toughness: Effects of void volume fraction, void shape and void distribution, International Journal of Solids and Structures 42 (18-19) (2005) 5097-5117. doi:10.1016/J. I JSOLSTR. 2005.02.028.

URL https://www.sciencedirect.com/science/article/pii/ S0020768305000892\#fig1

[40] F. Lipperman, M. Ryvkin, M. B. Fuchs, Crack arresting low-density porous materials with periodic microstructure, International Journal of Engineering Science 46 (6) (2008) 572-584. doi:10.1016/j. ijengsci. 2008.01 .004$. 